\title{
Photocatalytic removal of $17 \alpha$-ethinylestradiol (EE2) and levonorgestrel (LNG) from contraceptive pill manufacturing plant wastewater under UVC radiation
}

\author{
Deniz Nasuhoglu, Dimitrios Berk, Viviane Yargeau* \\ Department of Chemical Engineering, McGill University, 3610 University Street, Wong Building, H3A 2B2, Montreal, Quebec, Canada
}

\section{A R T I C L E I N F O}

\section{Article history:}

Received 28 September 2011

Received in revised form

27 December 2011

Accepted 3 January 2012

\section{Keywords:}

$17 \alpha$-Ethinylestradiol

Levonorgestrel

Photocatalysis

Photolysis

Pharmaceutical wastewater

\begin{abstract}
A B S T R A C T
Photolytic and photocatalytic removals of $17 \alpha$-ethinylestradiol (EE2) and levonorgestrel (LNG) in pharmaceutical wastewater were investigated under UVC radiation. Wastewater collected from WYETH, St-Laurent, Canada contained high concentrations of EE2 and LNG in suspension and coloring agent tartrazine in solution. Aqueous phase removals of EE2 and LNG were studied as individual contaminants in water and in complex matrices including: co-contaminants in water, in simulated synthetic wastewater and in the wastewater. After 30 min of UVC photocatalysis of the individual contaminants, removal efficiencies of EE2 and LNG were 92\% and 97\%, respectively, while higher photolytic removal was observed for LNG (94\%) compared to EE2 (60\%). Hydroxyl radicals were shown to contribute significantly to the removal of both compounds in water. In contrast to EE2, photolytic removal of LNG was higher than its photocatalytic removal efficiencies in all complex matrices. Higher photolytic removal of LNG was attributed to the fact that it absorbs UVC radiation considerably more than EE2. Lower photocatalytic removals of LNG in complex matrices compared to its photocatalytic removal as an individual contaminant was due to the presence of EE2 at concentrations up to five times larger than LNG in water, thus leading to increased competition for hydroxyl radicals and retarding LNG removal. In the wastewater matrix photocatalytic removals for EE2 and LNG were similar at 48\%, whereas the photolytic removal of LNG (76\%) was higher than EE2 (29\%). The applicability of UVC processes for reduction of hormone content in similar wastewaters was demonstrated.
\end{abstract}

(c) 2012 Elsevier B.V. All rights reserved.

\section{Introduction}

The occurrence of natural and synthetic chemicals in the aquatic environment has been reported regularly in the recent years [1,2] and the observed adverse effects of these compounds on human and aquatic wildlife by interfering with the endocrine system is now an issue of global concern [3,4]. These compounds are referred to as endocrine disrupting chemicals (EDCs). The U.S. Environmental Protection Agency (EPA) defines environmental EDCs as xenobiotics that interfere with the synthesis, secretion, transport, binding, action, or elimination of natural hormones in the body that are responsible for the maintenance of homeostasis, reproduction, development, and/or behaviour [5]. Common EDCs include natural estrogens such as estrone (E1), 17 $\beta$-estradiol (E2) and estriol (E3), synthetic estrogens such as $17 \alpha$-ethinylestradiol (EE2) and industrial chemicals such as bisphenol A, DDT, alkylphenols, PCBs and phthalic esters.

A major source of aquatic contamination by EDCs is the effluent of sewage treatment plants (STPs) associated with domestic and

\footnotetext{
* Corresponding author. Tel.: +1 514398 2273; fax: +1 5143986678.

E-mail address: viviane.yargeau@mcgill.ca (V. Yargeau).
}

hospital wastewater as well as with manufacturing plant wastewater. Estrogenic hormones have been commonly detected in the effluents of STPs, surface waters and even in treated drinking waters [2,6-8]. Among these, EE2 is a synthetic estrogen widely used in oral contraceptives and hormone replacement therapy. A whole lake addition study performed in Canada showed that spiking a lake with EE2 concentrations of 5-6 ng/L resulted in extinction of whole fish populations [9]. EE2, even at ng/L concentration levels, was also shown to induce the expression of vitellogenin in male fish, cause sex differentiation, and lead to the reduction in fish fertility $[10,11]$. LNG is a synthetic progestogen (i.e. progestin), used either alone or in combination with EE2 in a variety of hormonal formulations $[12,13]$. Occurrence of progestins only recently received attention, and currently there are very few data on the presence of these compounds in aquatic environments [14,15]. LNG was found to affect male fertility and exposure to high levels of LNG ( $500 \mu \mathrm{g} /$ day over 10 weeks) was shown to lead to azoospermia (no measurable sperm in semen) by Bebb et al. [16]. Due to the undesirable affects of EDCs on the environment and low removal in wastewater treatment plants, more effective treatment methods are necessary to mitigate their impact on the environment and public health.

Advanced oxidation processes (AOPs) have been recently investigated as complementary or alternative methods to conventional 
wastewater treatment. AOPs such as ozonation [17-19], fenton and photo-fenton oxidation [20,21], photocatalysis and photolysis [22-32] have been investigated as effective methods for elimination of estrogenic activity of EDCs such as EE2. Few photo-removal studies for EE2 were performed in complex matrices as mixtures of estrogens [25], with co-pollutants in complex water matrices $[23,24]$ and in natural waters [32]. Generally there is lack of data on the photo-removal of EE2 in a wastewater matrix, especially no information on removal of mixture estrogens and progestins in manufacturing plant wastewater is available. Additionally, there are no reported data on the photolytic or photocatalytic removal of LNG.

Based on the previously mentioned gap of knowledge, the objective of this work was to evaluate the applicability of UVC photolysis and photocatalysis to remove simultaneously EE2 and LNG in pure water matrix, and more importantly in a complex wastewater matrix produced at a pharmaceutical processing plant. The wastewater selected for this study was the highly concentrated wastewater generated from the oral contraceptive production facilities of WYETH, St-Laurent production plant. The current method of disposal of the first wash of the mixing vessels used for production is segregation followed by incineration. Incineration of large quantities of diluted aqueous solutions is costly. Therefore studying the applicability of a photolytic or a photocatalytic process as a wastewater treatment alternative is necessary to help reduce (or completely remove) the concentrations of the hormonally active compounds from the first wash, which might then be safely mixed with the rest of the wastewater generated at the plant.

\section{Materials and methods}

\subsection{Reagents}

$17 \alpha$-Ethinylestradiol (EE2, $\mathrm{C}_{20} \mathrm{H}_{24} \mathrm{O}_{2}, \geq 98 \%$ ), $17 \beta$-estradiol (E2, $\mathrm{C}_{18} \mathrm{H}_{24} \mathrm{O}_{2}, \geq 98 \%$ ), levonorgestrel (LNG, $\mathrm{C}_{21} \mathrm{H}_{28} \mathrm{O}_{2}, \geq 99 \%$ ), 19-norethindrone (NOR, $\mathrm{C}_{20} \mathrm{H}_{26} \mathrm{O}_{2}, \geq 99 \%$ ), tartrazine (TART, $\mathrm{C}_{16} \mathrm{H}_{9} \mathrm{~N}_{4} \mathrm{Na}_{3} \mathrm{O}_{9} \mathrm{~S}_{2}, \geq 98 \%$ ) and ammonium acetate ( $\geq 98 \%$ ) were obtained from Sigma-Aldrich, Canada. Commercial $\mathrm{TiO}_{2}$ Degussa P25 (70\% anatase and 30\% rutile) was used as catalyst with an average particle size of $30 \mathrm{~nm}$ and a BET surface area of $50 \mathrm{~m}^{2} / \mathrm{g}$, according to the manufacturer. HPLC grade methanol, acetonitrile, chloroform and isopropanol as well as $\mathrm{KOH}$ pellets were purchased from Fisher Scientific, Canada. 95\% ethanol was obtained from (Commercial Alcohols Inc., Boucherville). All the chemicals were used as received without purification.

\subsection{Wastewater collection and quantification of pharmaceutical compounds}

Industrial wastewater samples were collected at the WYETH production plant located in St-Laurent, Canada on November 11th, 2008 by taking a grab sample $(10 \mathrm{~L})$ from the first wash of the vessels used for production of oral contraceptive pills. The sample was collected in amber bottles and frozen within $2 \mathrm{~h}$ following sampling. The wastewater was stored at $-30^{\circ} \mathrm{C}$ until the time of treatment (12-18 months). The only information disclosed by the company about the constituents used in the production of the pills was the presence of EE2, LNG and dye FD\&C YELLOW 5 LAKE 960 (i.e. tartrazine). Visual observations indicated that a considerable amount of material was in suspension. The low solubility of EE2 and LNG suggests that a significant portion of the hormones were in suspension.

Aqueous phase EE2 and LNG concentration in the wastewater was determined by HPLC analysis of syringe filtered wastewater samples. In order to quantify the total hormone concentration
Table 1

Extraction recoveries of compounds of interest and their respective internal standards.

\begin{tabular}{lrrr}
\multicolumn{4}{l}{ Extraction recoveries (\%) } \\
\hline E2 & EE2 & NOR & LNG \\
\hline $97 \pm 4$ & $98 \pm 4$ & $101 \pm 5$ & $102 \pm 5$
\end{tabular}

(in suspension and in solution), a chloroform extraction method was developed. The optimum volumetric ratio of sample to chloroform was determined by sequentially adding $50 \mathrm{~mL}$ chloroform to a $100 \mathrm{~mL}$ of wastewater sample in a $250 \mathrm{~mL}$ separatory funnel. After each $50 \mathrm{~mL}$ chloroform addition, UV-vis absorbance spectrum of the organic portion was determined (Thermo Scientific Evolution 300). The optimum ratio was determined when negligible UV absorption in the $200-400 \mathrm{~nm}$ range was measured. This value was determined to be 5:1 (chloroform volume:sample volume). For the determination of extraction recoveries of EE2 and LNG, E2 and NOR were chosen as internal standards, respectively. In order to validate the extraction method and choice of internal standards, $250 \mathrm{~mL}$ pure reverse osmosis (RO) water samples were spiked with EE2, E2, NOR and LNG stock solutions in methanol at concentrations of $10 \mathrm{mg} / \mathrm{L}$ for each compound. Six $10 \mathrm{~mL}$ samples were extracted with $50 \mathrm{~mL}$ of chloroform in a $125 \mathrm{~mL}$ separatory funnel. $4 \mathrm{~mL}$ samples from the organic phase were withdrawn, chloroform was evaporated from the extracted samples by Thermo Scientific Savant SPD 131 DDA Speedvac Concentrator equipped with RVT 4104 refrigerated vapor trap, and the samples were reconstituted in $4 \mathrm{~mL}$ methanol to be analyzed by HPLC. The extraction recoveries of internal standards and respective pharmaceuticals of interest were shown to be satisfactorily close (Table 1 ), validating the extraction method and choice of internal standards. The described extraction method was applied to wastewater for the determination of the total concentrations of EE2 and LNG in the wastewater (as well as their total concentrations in pure suspensions). The concentration of coloring agent in the wastewater, tartrazine (TART) was quantified by UV-vis absorbance at $428 \mathrm{~nm}$. Since TART is strongly hydrophilic, transfer of this compound to the organic phase during extraction was not a concern. The aqueous portion of the wastewater sample was directly compared to standards of TART in RO water to quantify its concentration.

\subsection{Preparation of hormone stock solutions and types of matrices}

Stock solutions of EE2, E2, LNG and NOR (1000 mg/L) were prepared in methanol. EE2 and LNG stock solutions in methanol were used to prepare HPLC standards and solutions in RO water. Stock solutions of E2 and NOR, used as performance surrogates, were used to spike samples prior to chloroform extraction. All solutions were kept at $4{ }^{\circ} \mathrm{C}$ in the dark until the time of analysis (maximum time of storage was one week).

Photolytic and photocatalytic removal of EE2 and LNG were studied in four different matrices: (1) individual contaminants in RO water (Pure), (2) co-contaminants in RO water (MIX), (3) multicomponent mixture in tap water along with TART to obtain a simplified synthetic wastewater (SWW) and (4) the real wastewater from WYETH (WW). In order to compare the removal of EE2 and LNG in the four matrices studied, similar initial concentrations of compounds in all matrices were required. The total concentration of LNG in the WW was found to be above $500 \mathrm{mg} / \mathrm{L}$. Therefore, to ease the analysis and to reduce reaction times and the significantly high costs associated with hormonal compounds at the relevant wastewater concentrations, the degradation experiments were performed using 10 times diluted wastewater (first wash) and corresponding total concentrations in the other 
Table 2

Concentration of EE2 and LNG in the matrices studied.

\begin{tabular}{|c|c|c|c|c|c|}
\hline \multirow[t]{2}{*}{ Matrices } & \multirow[t]{2}{*}{$\mathrm{pH}$} & \multicolumn{2}{|c|}{ Total conc $(\mathrm{mg} / \mathrm{L})$} & \multicolumn{2}{|c|}{ Aqueous conc (mg/L) } \\
\hline & & EE2 & LNG & EE2 & LNG \\
\hline Pure EE2 & 5.5 & $\sim 5$ & - & $3.5-5$ & - \\
\hline Pure LNG & 5.5 & - & $\sim 50$ & - & $0.8-1.6$ \\
\hline MIX & 5.3 & $\sim 5$ & $\sim 50$ & $3.5-5$ & $0.8-1.6$ \\
\hline SWWa & 7.1 & $\sim 5$ & $\sim 50$ & $3.5-5$ & $0.8-1.6$ \\
\hline $\mathrm{WW}^{\mathrm{b}}$ & 6.8 & $\sim 5$ & $\sim 50$ & $1.6-2.4$ & $1.5-2.5$ \\
\hline
\end{tabular}

a In tap water contains TART at $0.7 \mathrm{mg} / \mathrm{L}$.

b Wastewater diluted with tap water contains TART at $0.7 \mathrm{mg} / \mathrm{L}$ and other unknown organic material.

matrices (EE2 $\sim 5 \mathrm{mg} / \mathrm{L}$ and $\mathrm{LNG} \sim 50 \mathrm{mg} / \mathrm{L}$ ). The concentrations of LNG and EE2 in the matrices studied are tabulated in Table 2. The working volumes were stirred overnight and sonicated for $30 \mathrm{~min}$ prior to treatment in order to obtain a well-mixed suspension and avoid agglomeration.

\subsection{Photolysis and photocatalysis setup}

Irradiation experiments were carried out in 2-L cylindrical water-cooled jacketed pyrex photoreactor $(215 \mathrm{~mm}$ height, $108 \mathrm{~mm}$ diameter). The reactor walls were covered with aluminum foil to avoid exposure to UV radiation. $1.6 \mathrm{~L}$ of working solution was charged to the reactor for each experiment. The solution was irradiated by a $\mathrm{Hg}-\mathrm{Ar}$ (Germicidal UV-C) lamp (Atlantic Ultraviolet Corp. GPH212T5L) located in the center of the reactor and protected in a quartz sleeve (maximum output at $254 \mathrm{~nm}$ ). It was previously shown that light intensity inside the reactor highly varied by position and the maximum intensity of incident radiation per unit volume was $1.3 \times 10^{-3} \pm 0.3$ Einstein/min/L [33]. Mixing was achieved by magnetic stirring, and oxygen was supplied via bubbling air through a sparger located at the bottom of the reactor. The concentration of $\mathrm{TiO}_{2}$ was fixed at $0.2 \mathrm{~g} / \mathrm{L}$ for all photocatalytic experiments. For photocatalytic experiments, $\mathrm{TiO}_{2}$ suspensions were sonicated for $30 \mathrm{~min}$ prior to addition to the reaction mixture to avoid agglomeration and subsequent reduction in active surface area. The initial $\mathrm{pH}$ for all the matrices studied is provided in Table 2. EE2 and LNG have high pKa values of 10.4 and 19.3, respectively [34]. At the studied ambient $\mathrm{pH}$ values they are not charged therefore slight changes in $\mathrm{pH}$ is expected not to affect their removal.

It was found that both photolytic and photocatalytic removal of EE2 and LNG in each matrix followed pseudo-first order reaction kinetics in the first $10 \mathrm{~min}$ of removal. Therefore the rate constants where determined from

$\ln \left(C / C_{o}\right)=-k t$

where, $C$ is the concentration of compound of interest $(\mathrm{mg} / \mathrm{L}), C_{0}$ is initial concentration $(\mathrm{mg} / \mathrm{L}), t$ is time ( $\mathrm{min}$ ) and $k$ is the pseudo-first order reaction rate constant $\left(\mathrm{min}^{-1}\right)$. Pseudo-first order reaction rate constants were calculated from the slope of the plots of $-\ln \left(C / C_{0}\right)$ against time.

Adsorption and dark control experiments were performed in triplicates to make sure that removal of compounds was not due to adsorption of compounds to either $\mathrm{TiO}_{2}$ particles or to the walls of the reactor. Suspensions of EE2 (5 mg/L) and LNG $(50 \mathrm{mg} / \mathrm{L})$ as well as aqueous solutions of TART $(0.7 \mathrm{mg} / \mathrm{L})$ were tested for adsorption onto $\mathrm{TiO}_{2}$ at a fixed concentration of $0.2 \mathrm{~g} / \mathrm{L}$. Erlenmeyer flasks were filled with $\mathrm{TiO}_{2}$ and the compounds of interest were placed inside an incubator shaker set at $25^{\circ} \mathrm{C}$. After $24 \mathrm{~h}$, samples were analyzed by HPLC and compared to the controls containing only the compounds. Dark control experiments for all compounds were performed inside the reactor with the lamp turned off. Samples were taken over a period of $2 \mathrm{~h}$ and analyzed for EE2 and LNG content to see if any removal was due to sampling or normal operation of the reactor. Samples were analyzed for concentrations of LNG and EE2, both as total and in solution.

Evidence for hydroxyl radical participation in removal of both EE2 and LNG in aqueous phase was evaluated by scavenging experiments. The method of scavenging and the concentrations of scavenging compounds were based on the results reported by Palominos et al. [35]. Isopropanol has been described as one of the best hydroxyl radical quencher due to its high reaction rate constant with the radical $\left(1.9 \times 10^{9} \mathrm{~mol} / \mathrm{L} / \mathrm{s}\right)$ [36]. In this work, scavenging of hydroxyl radicals was achieved by adding isopropanol to the reaction mixture at a molar concentration which was three orders of magnitude larger than the initial total molar concentration of EE2 and LNG.

\subsection{Light intensity determination and fraction of light absorbed}

In order to quantify the intensity of incident radiation, azoxybenzene was used as a chemical actinometer. The method was modified from the technique developed by Bunce et al. [37]. The detailed description of the actinometric method employed here can be found in a previous study [33]. All the actinometric experiments were performed at a single location in the reactor (radial distance of $2 \mathrm{~cm}$ away from the lamp, at height of $10 \mathrm{~cm}$ from the bottom of the reactor). Maximum available light intensity $\left(I_{\max }\right)$ was determined from the amount of light absorbed by the actinometric solution when the reaction system is only composed of pure RO water (no hormones, etc.) The actinometric solution was irradiated in each matrix (i.e. pure EE2, pure LNG, MIX, SWW and WW) and the associated light intensity was measured $\left(I_{\mathrm{a}}\right)$. The differences between $I_{\max }$ and $I_{\mathrm{a}}$ were normalized by $I_{\max }\left(\left[I_{\max }-I_{\mathrm{a}}\right] / I_{\max }\right)$ to give the respective fractions of light absorbed by the additional constituents of the matrices studied.

\subsection{Analytical methods}

Prior to analysis, samples were filtered using $0.22 \mu \mathrm{m}$ syringe filters. EE2 and LNG concentrations were monitored by a HPLC system (Agilent 1200 series) equipped with a Zorbax Eclipse Plus C-8 (Agilent, $4.6 \mathrm{~mm} \times 150 \mathrm{~mm}, 3.5$ microns) and two detectors (fluorescence and diode array) with an injection volume of $20 \mu \mathrm{L}$. Starting from initial conditions of acetonitrile $/ 5 \mathrm{mM}$ ammonium acetate $30 / 70(\mathrm{v} / \mathrm{v})$, the mobile-phase gradient linearly increased to $60 / 40(\mathrm{v} / \mathrm{v})$ over $20 \mathrm{~min}$. The flow rate was set at $0.8 \mathrm{~mL} / \mathrm{min}$. The same HPLC method was used for samples in water and in methanol. The excitation and emission wavelengths of the fluorescence detector were respectively set at $280 \mathrm{~nm}$ and $310 \mathrm{~nm}$ to quantify EE2 concentration. For the LNG concentration the diode array detector was set at a wavelength of $244 \mathrm{~nm}$. Chemical oxygen demand (COD) of the wastewater sample was measured by using a HACH Digital Reactor Block (DRB 200), a HACH spectrophotometer (DR/2500) and low range (3-150 mg/L) COD digestion vials (HACH). Total organic content (TOC) determination of the wastewater was achieved via Shimadzu TOC-VCPH total organic carbon analyzer.

\section{Results and discussion}

\subsection{Quantification of the pharmaceuticals in the wastewater}

The method of determining the required volumetric ratio of chloroform to sample was described in Section 2.2. The evolution of UV-vis chromatograms associated to the described procedure is presented in Supplementary Fig. S1. The volumetric ratio of sample to chloroform was determined to be $1: 5$ to maximise the recovery 
and obtain an accurate measurement of the initial concentration of EE2 and LNG in the wastewater.

The total (suspended and dissolved) concentrations of EE2 and LNG in the wastewater were determined to be $55.1 \pm 7.5$ and $567 \pm 25 \mathrm{mg} / \mathrm{L}$, respectively. The concentration of the coloring agent, TART, in the wastewater was $7.2 \pm 0.5 \mathrm{mg} / \mathrm{L}$ by UV-vis absorption analysis at $428 \mathrm{~nm}$. COD and TOC measurements were made in a 1000 times diluted version of the wastewater due to its highly concentrated state. Initial COD and TOC values were $78,600 \pm 1600 \mathrm{mg} / \mathrm{L}$ and $28,600 \pm 7100 \mathrm{mg} / \mathrm{L}$, respectively.

The characterization results suggest that the wastewater sample obtained from WYETH is highly concentrated both in hormones and other undisclosed constituents. The high COD and TOC values can be attributed to the presence of organic compounds used in the preparation of the contraceptive pills that also end up in the first wash of the production vessels. As mentioned previously the degradation experiments were performed using 10 times diluted wastewater (first wash), the resulting corresponding total and aqueous concentrations are tabulated in Table 2. All adsorption and degradation experiments were performed at these initial total and respective aqueous phase concentrations. Where applicable the total concentration refers to the total hormone content including both in suspension and in solution, as measured after chloroform extraction (described in Section 2.2).

\subsection{Adsorption and control experiments}

After $24 \mathrm{~h}$ of mixing, adsorption of $14.8 \pm 4.7 \%, 2.1 \pm 0.5 \%$ and $4.4 \pm 1.2 \%$ were observed for TART, EE2 and LNG, respectively. The total concentrations of EE2 and LNG obtained from experiments performed in the reactor in the absence of light, with or without $\mathrm{TiO}_{2}$, are shown in Fig. 1. No measurable loss of EE2 was observed suggesting that loss due to adsorption to the reactor wall is negligible (Fig. 1a). Although the LNG soluble concentration did not change (results not shown), the total concentration of LNG obtained by extraction was highly variable (Fig. 1b) and some of the concentrations measured were even higher than the initial total LNG concentration. These unexpected results are due to the nonhomogeneity of the solution in terms of LNG concentration because of its high concentration in solids. Deposition of suspended solids was also visually confirmed from the formation of a deposit on the quartz sleeve and the sides of the pyrex reactor. Because of these limitations, the LNG concentration was monitored only in the aqueous phase, which was stable throughout the experiments.

\subsection{Photolytic and photocatalytic removal of EE2, LNG and TART as pure compounds in $\mathrm{RO}$ water}

The removal data of TART in RO water is presented in Fig. 2a. Photocatalytic removal of TART is considerably higher than its photolytic removal. Complete elimination of color was achieved during photocatalytic treatment after 45 min while only $3 \%$ was removed by photolysis. A recently published study by Gupta et al. [38] also confirms the slower removal rate under photolytic conditions. In the work of Gupta et al. [38], the pseudo-first order reaction rate constant for photocatalysis at $0.2 \mathrm{~g} / \mathrm{L} \mathrm{TiO}_{2}$ concentration was estimated to be approximately $4.5 \times 10^{-2} \mathrm{~min}^{-1}$ compared to $7.8 \pm 1.7 \times 10^{-2} \mathrm{~min}^{-1}( \pm 1$ standard deviation) calculated in the work presented here. Even though the pseudo-first order reaction rate constant values are of the same order of magnitude, the discrepancy can be associated to various factors such as: the initial concentration of TART used by Gupta et al. [38] was two orders of magnitude higher than what was used here, the photocatalyst used was pure anatase $\mathrm{TiO}_{2}$ instead of Degussa P25 (anatase/rutile) powder and a smaller reactor was employed by Gupta et al. [38] $(150 \mathrm{~mL})$, leading to differences in light intensity distribution.
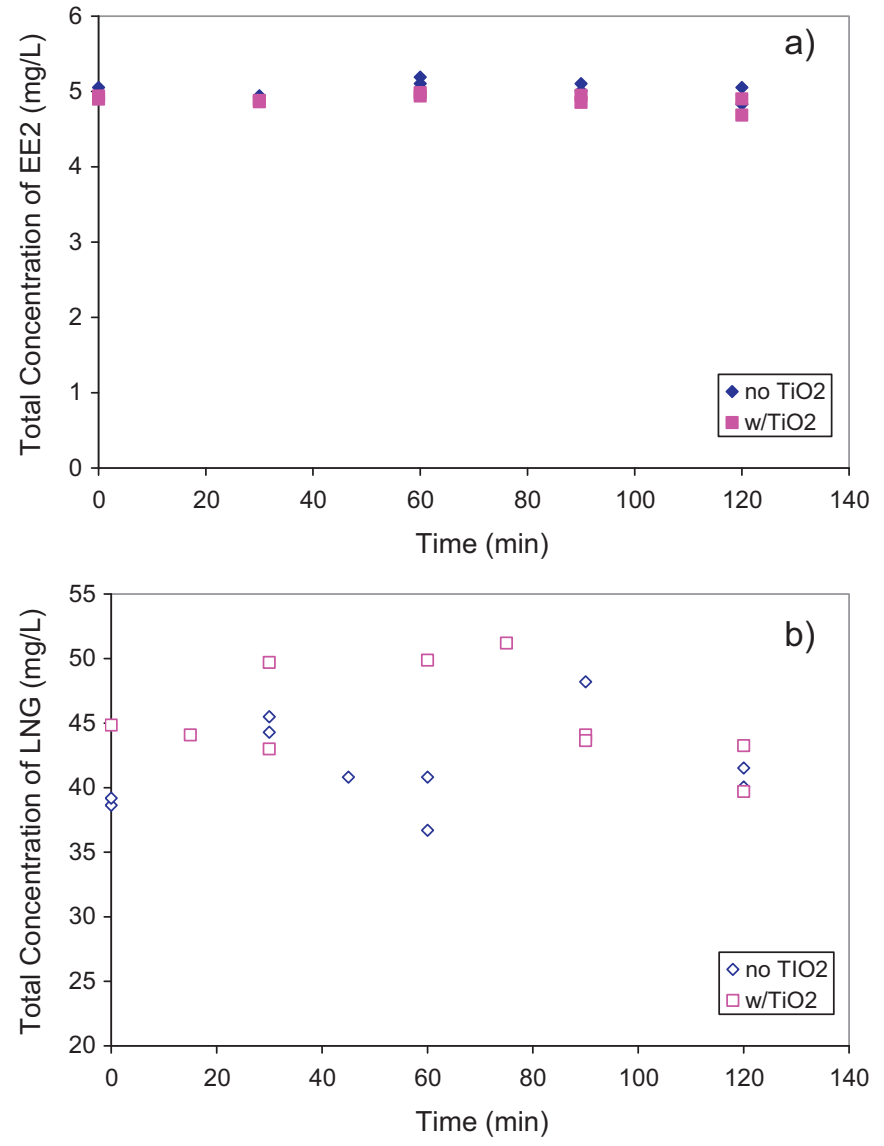

Fig. 1. Evolution of total concentration of (a) EE2 and (b) LNG under dark conditions in the reactor in a pure RO water matrix.

The removal data of EE2 as a single contaminant in RO water is presented in Fig. 2b (aqueous). EE2 removal is considerably enhanced in the presence of $\mathrm{TiO}_{2}$. After $30 \mathrm{~min}$ of irradiation, $92 \pm 7 \%$ of initial EE2 in the aqueous phase is removed during photocatalysis compared to $60 \pm 12 \%$ photolytic removal. The work by Liu et al. [26] for the photolytic removal under UVC radiation of EE2 resulted in 50\% removal over 30 min which is in accordance with the value presented here. From another study by Mazellier et al. [28], 40\% photolytic removal over $30 \mathrm{~min}$ was calculated from their reported data. This slightly lower removal can be attributed to the fact their working volume $(4 \mathrm{~L})$ was more than twice the working volume used in this work. Higher reactor volume would lead to larger variations in light distribution inside the reactor, resulting in decrease in light availability and thus inefficient removal. A more recent study, investigating the removal of estrogenic compounds in multi-component estrogen mixtures by Li Puma et al. [25], also confirmed faster removal of EE2 during photocatalysis than photolysis. They obtained a value of $86 \%$ photocatalytic removal of EE2 in a mixture of E1, E2 and E3 over 30 min, which lies within the removal efficiency reported here. However, they obtained a far lower removal efficiency of about $22 \%$ of EE2 under photolysis over 30 min compared to the photolytic removal presented here. The slower EE2 removal is most likely due to the presence of other estrogens in the multi-component mixture leading to enhanced competition and reducing light availability. The removal trend of total EE2 was shown to closely follow the trend observed for aqueous removal. Similar pseudo-first order reaction rate constants are calculated for total and aqueous phase removal of EE2 during both treatments (Table 3 ). This suggests that the system is mass-transfer 

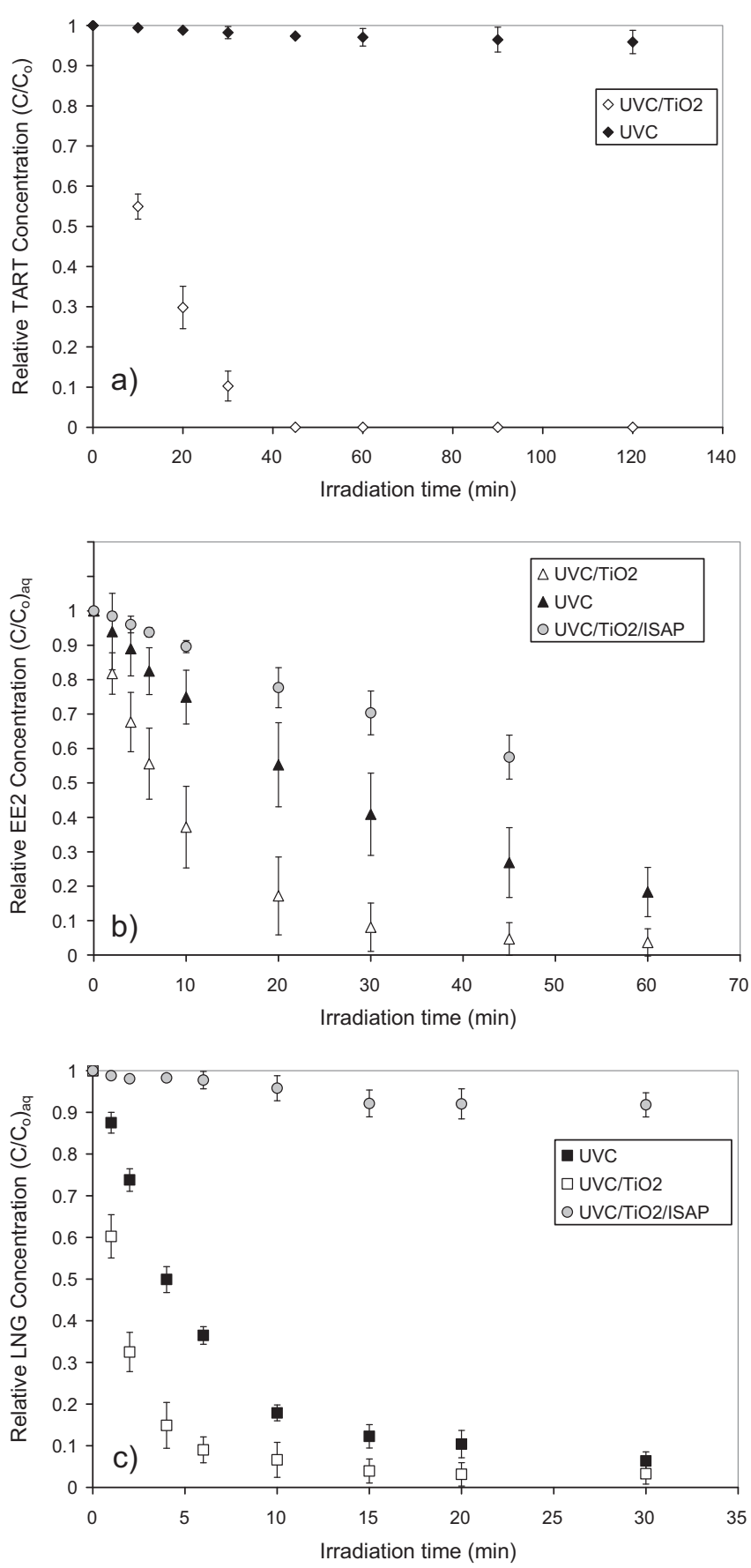

Fig. 2. Evolution of relative concentration of (a) TART (b) EE2 and (c) LNG during photolysis (UVC) and photocatalysis $\left(\mathrm{UVC} / \mathrm{TiO}_{2}\right)$ in pure RO water matrix (based on aqueous concentrations). Effect of the presence of hydroxyl radicals during photocatalysis on removal of (b) EE2 and (c) LNG is also studied by scavenging the hydroxyl radicals by addition of isopropanol. Errors bars $= \pm 1$ standard deviation.

Table 3

Pseudo-first order reaction rate constants of EE2 based on total and aqueous concentration for UVC photolysis and photocatalysis.

\begin{tabular}{lll}
\hline & \multicolumn{2}{l}{ Pseudo first order reaction rate constant, $k \times 10^{-2}\left(\mathrm{~min}^{-1}\right)$} \\
\cline { 2 - 3 } & Based on aqueous concentration & Based on total concentration \\
\hline $\mathrm{UVC}$ & $3.0 \pm 1.3$ & $2.8 \pm 1.1$ \\
$\mathrm{UVC} / \mathrm{TiO}_{2}$ & $10.6 \pm 5.6$ & $9.5 \pm 3.2$ \\
\hline
\end{tabular}

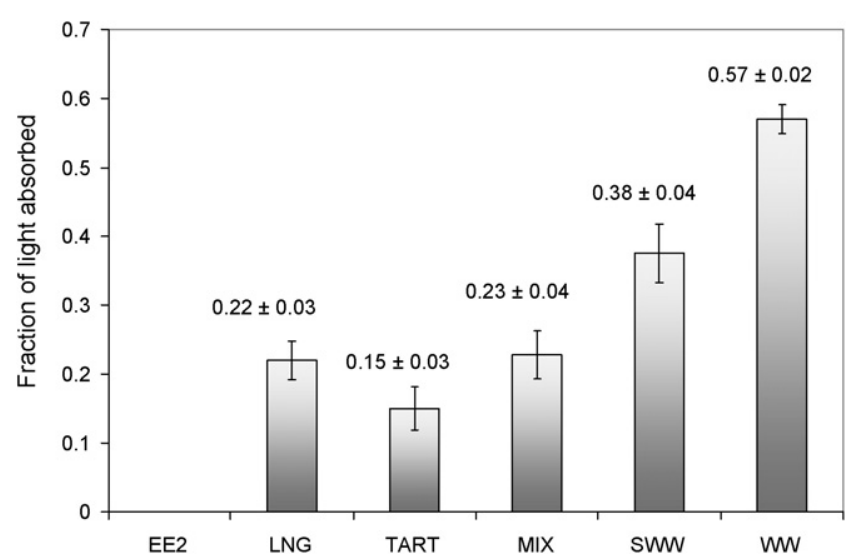

Fig. 3. Fraction of light absorbed by each compound and by each matrix determined using azoxybenzene actinometry. Errors bars $= \pm 1$ standard deviation.

limited, i.e. the removal rates in the aqueous phase are higher than the dissolution rate of EE2 for both photolysis and photocatalysis.

Preliminary experiments indicated that the total amount of LNG removed during treatment lies in the range of the error of measurement of the total concentration of LNG. Considering this limitation, only aqueous phase degradation of LNG was considered here. Removal data of LNG in RO water are presented in Fig. 2c. Similarly to EE2, removal of LNG during photocatalytic treatment was faster than during photolysis. Over $10 \mathrm{~min}$ of irradiation, $93 \pm 2 \%$ of initial LNG present in solution is removed during photocatalysis compared to $82 \pm 4 \%$ photolytic removal. However, in both cases LNG is no longer detected after $30 \mathrm{~min}$. The fact that more than $80 \%$ of LNG was removed within 10 min compared to about $25 \%$ removal observed for EE2 for the same time period strongly suggests that LNG is far more sensitive to UVC radiation than EE2. Molar absorption coefficients $(\varepsilon)$ at $254 \mathrm{~nm}$ of EE2 and LNG $\left(\varepsilon_{\mathrm{EE} 2}=216 \pm 40 \mathrm{~L} / \mathrm{mol} / \mathrm{cm}, \varepsilon_{\mathrm{LNG}}=8617 \pm 210 \mathrm{~L} / \mathrm{mol} / \mathrm{cm}\right)$ calculated from UV-vis spectra shown for both compounds in Supplementary Fig. S2 also support this observation. Additionally, the inhibition of photocatalytic removal for both compounds was observed when hydroxyl radicals were scavenged (Fig. $2 \mathrm{~b}$ and c). After $30 \mathrm{~min}$ of irradiation, $62 \%$ and $88 \%$ reduction in photocatalytic removal efficiencies for EE2 and LNG, respectively. This observation suggests that the hydroxyl radicals contribute strongly towards the degradation of compounds during photocatalysis.

\subsection{Removal of EE2 and LNG in complex matrices}

Fig. 3 shows the fractions of light absorbed by each compound and by each matrix. Compared to LNG and TART, the presence of EE2 did not lead to any measurable decrease in the available light, suggesting that EE2 has minimal absorption of radiation at $254 \mathrm{~nm}$ (also confirmed from UV-vis spectrum for EE2 in Supplementary Fig. S2). LNG and TART alone absorb $22 \%$ and $15 \%$ of the max light available, respectively. The fact that WW matrix absorbs higher fraction of light when compared to that by SWW confirms the possibility of other unidentified species contributing to the light absorption and resulting decrease in light available for EE2 and LNG to undergo direct photolysis in complex WW matrix. When $0.2 \mathrm{~g} / \mathrm{L}$ of $\mathrm{TiO}_{2}$ is present in the system, $97 \%$ of the light is absorbed. Thus, for the photocatalytic experiments the availability of light is already diminished and the contribution of photolysis to the removal of any compound during photocatalytic treatment can be considered minimal.

Fig. 4 shows the photolytic removals of EE2 (Fig. 4a) and LNG (Fig. 4b) in all three matrices (Pure, MIX and SWW). Pseudo-first order reaction rate constants calculated are tabulated in Table 4. 

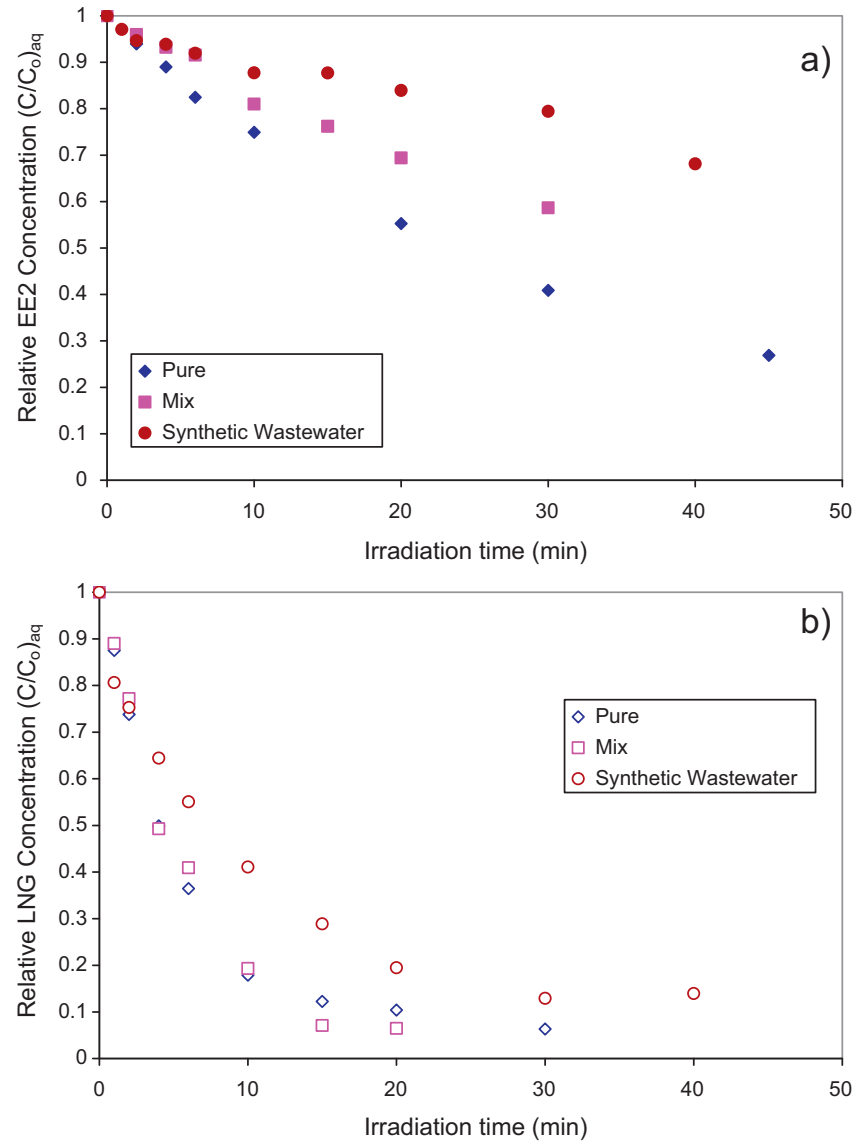

Fig. 4. Evolution of relative EE2 (a) and relative LNG (b) concentration during photolysis (based on aqueous concentrations) in Pure, MIX and SWW systems.

As the complexity of the matrix increases (from Pure to SWW), the general trend for both compounds is that the removal efficiency decreases. At 30 min of irradiation, EE2 the photolytic removal efficiency was $60 \%$ as pure compound, where as this value is decreased to $41 \%$ and $21 \%$, for MIX and SWW, respectively. For LNG, in pure and MIX systems photolytic removal efficiency after $30 \mathrm{~min}$ was measured to be $94 \%$, however this value reduced to $87 \%$ for the SWW system. This reduction of removal efficiency and rate is expected, since presence of other organic species and suspended material can compete for the photons or lead to scattering of light both resulting in the reduction of available light for the compound of interest to go through direct photolysis.

In all three matrices photolytic removal efficiencies and rates are higher for LNG compared to EE2. For EE2, going from pure to MIX, about $40 \%$ reduction in removal rate is observed, however for LNG the removal rates and efficiencies are not reduced in the MIX system. The presence of EE2, does not have an impact on the

Table 4

Pseudo-first order reaction rate constants of EE2 and LNG during their photolytic and photocatalytic removals in all matrices.

\begin{tabular}{|c|c|c|c|c|}
\hline \multirow[b]{3}{*}{ Matrix } & \multicolumn{4}{|c|}{ Pseudo first order reaction rate constant, $k \times 10^{-2}\left(\mathrm{~min}^{-1}\right)$} \\
\hline & \multicolumn{2}{|l|}{ EE2 } & \multicolumn{2}{|l|}{ LNG } \\
\hline & UVC & $\mathrm{UVC} / \mathrm{TiO}_{2}$ & UVC & $\mathrm{UVC} / \mathrm{TiO}_{2}$ \\
\hline PURE & $3.0 \pm 1.3$ & $10.8 \pm 5.6$ & $17.4 \pm 1.1$ & $40.3 \pm 4.1$ \\
\hline MIX & $1.8 \pm 0.4$ & $10.9 \pm 3.1$ & $17.6 \pm 0.9$ & $8.4 \pm 0.9$ \\
\hline SWW & $1.1 \pm 0.2$ & $6.1 \pm 1.7$ & $7.8 \pm 0.4$ & $2.3 \pm 0.6$ \\
\hline $\mathrm{WW}^{\mathrm{a}}$ & $0.8 \pm 0.2$ & $2.2 \pm 1.1$ & $7.2 \pm 0.3$ & $2.1 \pm 0.5$ \\
\hline
\end{tabular}

a Initial aqueous phase concentrations of EE2 and LNG in WW are not the same as in the other matrices.
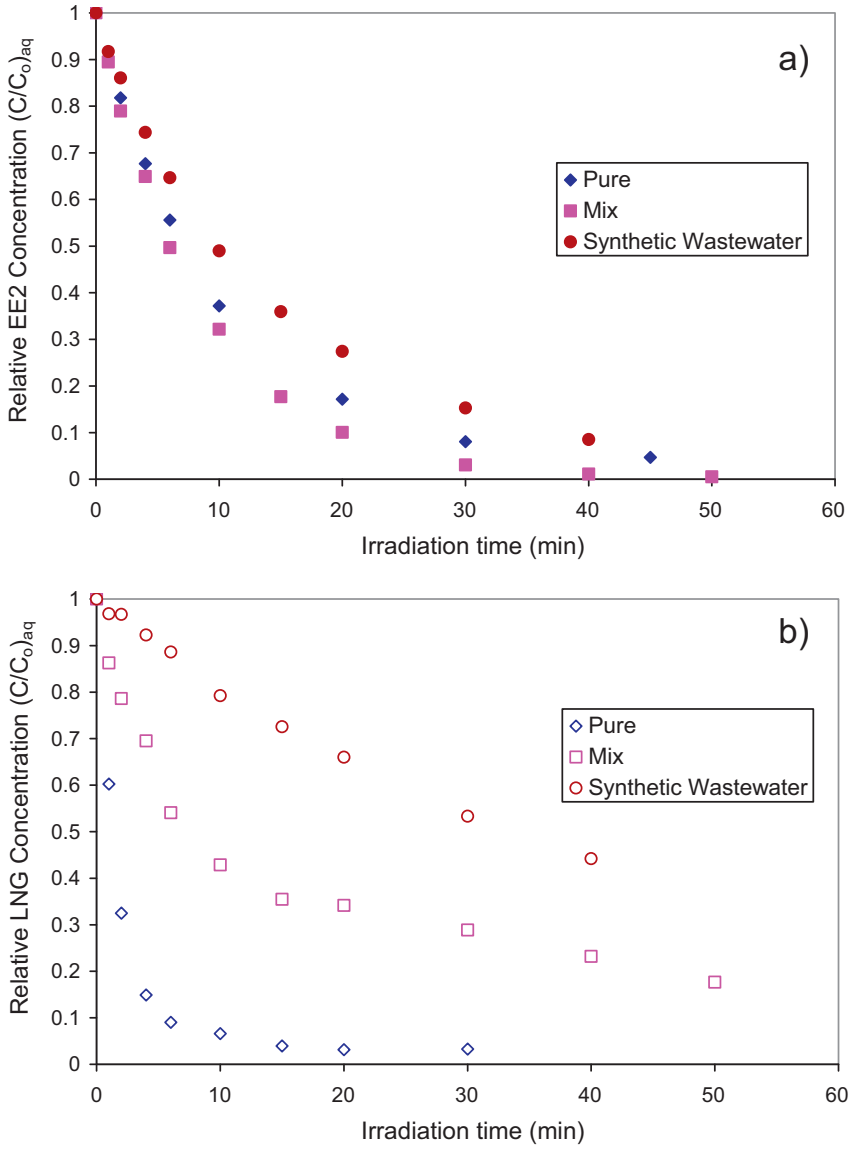

Fig. 5. Evolution of relative EE2 (a) and relative LNG (b) concentration during photocatalysis (based on aqueous concentrations) in Pure, MIX and SWW systems.

photolytic removal of LNG, but presence of LNG has a large impact on EE2 removal. This behaviour can be attributed to two aspects. First, in the MIX, the total LNG concentration is $40-60 \mathrm{mg} / \mathrm{L}$ where as EE2 concentration is only about $5 \mathrm{mg} / \mathrm{L}$. Since LNG is present at a considerably higher concentration than EE2, its presence will have a greater impact. Second and most importantly, LNG absorbs significantly higher than EE2 in the UVC range as observed from their respective UV-vis spectra (Supplementary Fig. S2) and from the fraction of light absorbed by pure LNG (Fig. 3). The amount of light available for EE2 removal in pure is considerably reduced by the addition of a high concentration compound with high UVC absorption due to scattering and competition effects. High photolytic removal rate associated to LNG in pure systems, is not hindered by the presence of EE2 due to its lower total concentration and considerably lower UVC absorption. The fact that the fraction of light absorbed by the MIX is almost entirely due to the presence of LNG also supports this observation. In all matrices, photolytic removal of LNG is slower after $30 \mathrm{~min}$ of irradiation compared to its initial removal rate. This can due to the accumulation of degradation products at prolonged irradiation resulting in enhanced absorption or scattering of light. Removal rates in the SWW are further reduced by $38 \%$ and $56 \%$ for EE2 and LNG, respectively compared to their removal rates in MIX. In SWW, the reduction in removal rate of LNG is more pronounced than that of EE2. As evidenced from Fig. 3, an additional $15 \%$ of light is absorbed in the SWW compared to MIX due to the presence of TART. Since LNG is more sensitive to direct photolysis than EE2 under UVC radiation, reduction in light availability affect the photolytic removal of LNG more than it does EE2. 
Fig. 5 shows the photocatalytic removals of EE2 (Fig. 5a) and LNG (Fig. 5b) in three matrices (Pure, MIX and SWW). The calculated pseudo-first order reaction rate constants are tabulated in Table 4. Similar to the trends observed for the photolytic removals of these compounds, their photocatalytic removals tend to decrease as the matrix gets more complex. At 30 min of irradiation, the photocatalytic removal efficiency of EE2 was $97 \%$ as pure compound, where as this value is decreased to $92 \%$, and $85 \%$ for MIX and SWW, respectively. For pure LNG, photocatalytic removal efficiency after $30 \mathrm{~min}$ was measured to be $97 \%$, however this value reduced to $71 \%$ and $47 \%$ for MIX and SWW, respectively. Hydroxyl radicals generated during photocatalysis have high oxidizing potential and they are considered to be less selective than a variety of oxidizing species. Therefore, overall reduction in removal efficiencies can be associated to the enhanced competition for hydroxyl radicals due to the presence of other organic species in more complex systems. This reduced photocatalytic removal of EE2 in a complex reaction medium was also reported by Karpova et al. [24] in presence of saccharose and urine. As mentioned earlier the total concentration of LNG (40-60 mg/L) is considerably larger than the total concentration of EE2 $(5 \mathrm{mg} / \mathrm{L})$; thus in the MIX system high reduction in removal rate would be expected for EE2 compared to the removal rate observed as pure compound. In contrast to our expectation, the photocatalytic removal rate of EE2 in the MIX system was not reduced whereas the LNG rate decreased considerably by $80 \%$ when compared to their respective pure compound photocatalytic removal rates. The explanation lies in the aqueous phase concentrations of the compounds. EE2 being more soluble than LNG, its aqueous concentration is up to five times higher than the LNG aqueous concentration (3.5-5 mg/L EE2 compared to $0.8-1.3 \mathrm{mg} / \mathrm{L} \mathrm{LNG}$ ). It is therefore more likely for hydroxyl radicals to encounter EE2 molecules than LNG. The slight increase in the aqueous concentration in the MIX due to the addition of LNG then had no considerable influence on the removal rate of EE2. However for LNG, in the MIX system the addition of a compound (EE2) at higher concentration induces more competition; thus the removal rate was extensively reduced. The previous observations also support the hypothesis that most of the photocatalytic reactions occur in the aqueous phase and that the suspended particles are not attacked by hydroxyl radicals within the irradiation time frame investigated here.

It was previously shown that removal of TART is mainly due to the generation of hydroxyl radicals during photocatalysis while removal by direct photolysis was minimal (Fig. 2a). This implies that the presence of this compound would contribute to consumption of hydroxyl radicals when present in a mixture. Due to the presence of TART, reduction of removal rates by $44 \%$ and $72 \%$ from MIX to SWW systems was observed for EE2 and LNG, respectively. EE2 still has the highest aqueous concentration in SWW, therefore it experiences competition of hydroxyl radicals to a lesser extent.

The WW samples used for UVC irradiation experiments had different aqueous phase concentrations from the samples used for quantification of hormones. The expected aqueous phase concentration of EE2 from the characterization tests was $5 \mathrm{mg} / \mathrm{L}$; however this value was $1.6-2.4 \mathrm{mg} / \mathrm{L}$ in the WW system. The expected aqueous phase concentration of LNG was $0.8-1.3 \mathrm{mg} / \mathrm{L}$, but the samples used for degradation experiments contained $1.5-2.5 \mathrm{mg} / \mathrm{L}$ of LNG. Even though, the real concentrations are of the same order of magnitude with the expected values, the ratio of aqueous phase concentrations of EE2 to LNG are different. Therefore, removals of EE2 and LNG in the WW system were studied separately, as comparison of removals in WW to removals in other matrices would not be possible. Removal data of EE2 and LNG in the WW system are presented in Fig. 6. The corresponding pseudo-first order reaction rate constants are tabulated in Table 4 . After 40 min of irradiation time, the removal efficiencies of EE2 in the WW system were 36\% and $59 \%$ during photolysis and photocatalysis, respectively. On the
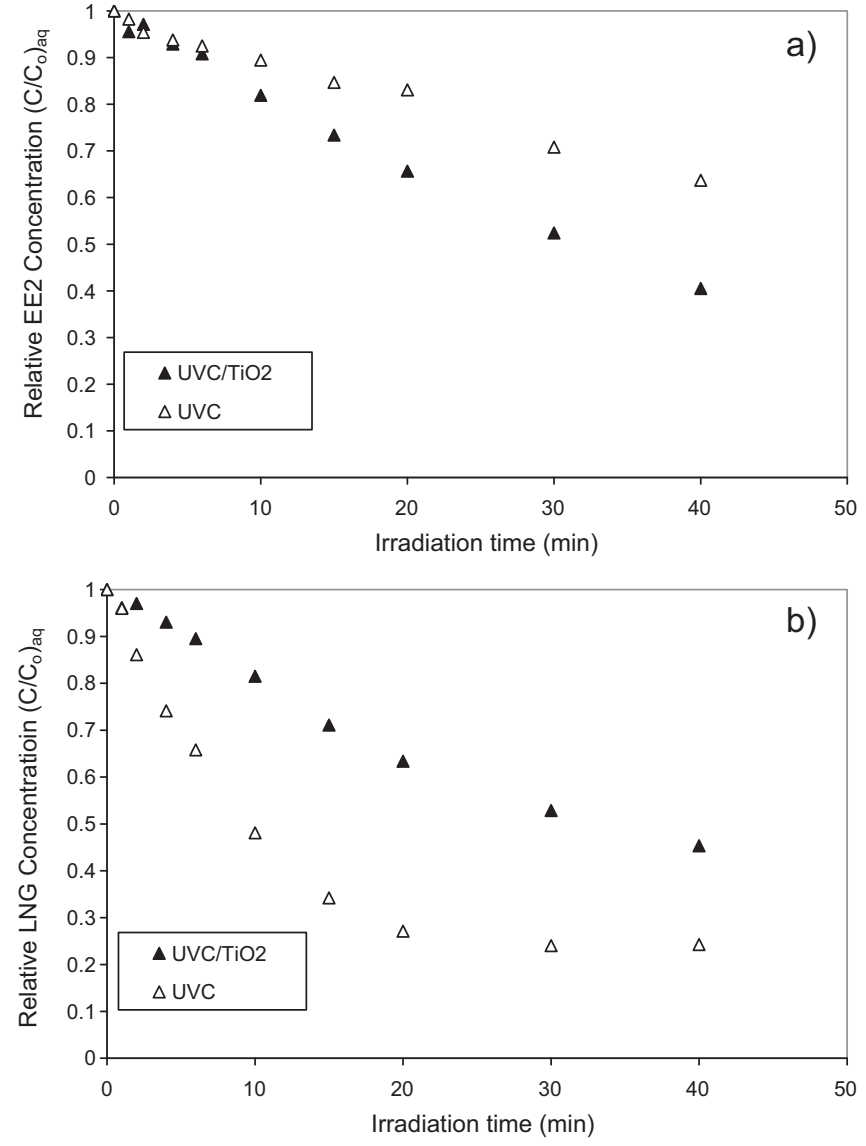

Fig. 6. Evolution of relative EE2 (a) and relative LNG (b) concentrations (based on aqueous concentrations) during photolysis (UVC) and photocatalysis $\left(\mathrm{UVC} / \mathrm{TiO}_{2}\right)$ in the WW system.

other hand, photolytic removal efficiency of LNG (76\%) in the WW system was larger than its photocatalytic removal efficiency (55\%). Given similar initial aqueous phase concentrations of EE2 and LNG, as is the case in the treated WW, photocatalytic removal efficiencies and rates of these compounds are similar since hydroxyl radicals are known to be non-selective oxidizing species. The higher sensitivity of LNG to direct degradation by UVC radiation leads to higher removal of this compound compared to EE2 during photolytic treatment.

Estrogenic activity of EE2 was shown to be completely removed by Coleman et al. [22] under both UVA photolysis and UVA photocatalysis with the latter being faster. Also Mazellier et al. [28] showed that the products generated from UVA photolysis of EE2 were not different than UVC photolysis. Conversely, Rosenfeldt et al. [29] demonstrated that during UVC photolysis estrogenic activity of EE2 was not reduced. In addition to these results, the mechanism of removal of EE2 during UVA photocatalysis is not expected to be different from that of UVC photocatalysis since for both treatment methods the generation of hydroxyl radicals is mainly responsible for the removal of the parent compound as also demonstrated here in Fig. $2 \mathrm{~b}$ and c. Therefore, during photocatalysis of EE2 the estrogenic activity is expected to be reduced. However for UVC photolysis more investigation about estrogenic activity removal is necessary to completely evaluate the possibility of using this as an alternative treatment method. Based on our results, if the objective of a treatment system is to remove the estrogenic activity due to EE2 in similar wastewaters, photocatalytic treatment would be recommended over photolytic treatment. For LNG, photolytic removal rates are higher in all complex matrices (MIX, SWW and WW). However, photolytic and photocatalytic 
degradation products of LNG might possess estrogenic activity. Therefore, future experiments should include the identification of degradation products of this compound and assess the hormonal activity of treated samples in order to compare the performances of photolysis and photocatalysis for removal of LNG.

\section{Conclusions}

The applicability of UVC photolysis and photocatalysis to industrial pharmaceutical wastewater was demonstrated for the removal of EE2 and LNG. Results related to photolytic and photocatalytic removals of LNG and its mixtures with EE2 and other wastewater components are presented here for the first time in the literature. Only LNG was shown to be completely removed by photolysis within the irradiation time frame studied here. Complete photocatalytic removals of both compounds as individual contaminants indicated that, UVC photocatalysis can be applied satisfactorily to similar wastewaters especially if these type of wastewaters are further diluted to solubility limits of hormonally active compounds to avoid mass transfer limitations. Similar removal efficiencies and removal rates were determined for EE2 and LNG in the WW system after 40 min of photocatalytic treatment. This suggested that simultaneous removal of both compounds in a complex matrix is possible during UVC photocatalysis. Only very recently, researchers started investigating the occurrence and environmental impact of progestins, especially LNG. Therefore, the results presented here provide researchers with strong evidence for applying UVC induced photodegradation processes to mitigate possible adverse environmental effects of this compound.

\section{Acknowledgments}

Authors would like to acknowledge the Natural Sciences and Engineering Research Council of Canada (NSERC) and Eugenie Ulmer Lamothe Chemical Engineering Fund (McGill University) for the financial support provided for this work and WYETH, StLaurent, Montreal for supplying the wastewater.

\section{Appendix A. Supplementary data}

Supplementary data associated with this article can be found, in the online version, at doi:10.1016/j.cej.2012.01.012.

\section{References}

[1] T.A. Hanselman, D.A. Graetz, A.C. Wilkie, Manure-Borne estrogens as potential environmental contaminants: a review, Environ. Sci. Technol. 37 (2003) 5471-5478.

[2] T.A. Ternes, M. Stumpf, J. Mueller, K. Haberer, R.D. Wilken, M. Servos, Behavior and occurrence of estrogens in municipal sewage treatment plants. I. Investigations in Germany, Canada and Brazil, Sci. Total Environ. 225 (1999) 81-90.

[3] T. Colborn, F.S. vom Saal, A.M. Soto, Developmental effects of endocrinedisrupting chemicals in wildlife and humans, Environ. Health Perspect. 101 (1993) 378-384.

[4] J.G. Vos, E. Dybing, H.A. Greim, O. Ladefoged, C. Lambré, J.V. Tarazona, I. Brandt A.D. Vethaak, Health effects of endocrine-disrupting chemicals on wildlife, with special reference to the European situation, Crit. Rev. Toxicol. 30(2000)71-133.

[5] V. Belgiorno, L. Rizzo, D. Fatta, C. Della Rocca, G. Lofrano, A. Nikolaou, V. Naddeo, S. Meric, Review on endocrine disrupting-emerging compounds in urban wastewater: occurrence and removal by photocatalysis and ultrasonic irradiation for wastewater reuse, Desalination 215 (2007) 166-176.

[6] C. Baronti, R. Curini, G. D’Ascenzo, A. Di Corcia, A. Gentili, R. Samperi, Monitoring natural synthetic estrogens at activated sludge sewage treatment plants and in a receiving river water, Environ. Sci. Technol. 34 (2000) 5059-5066.

[7] A.C. Belfroid, A. Van der Horst, A.D. Vethaak, A.J. Schäfer, G.B.J. Rijs, J. Wegener, W.P. Cofino, Analysis and occurrence of estrogenic hormones and their glucuronides in surface water and waste water in The Netherlands, Sci. Tota Environ. 225 (1999) 101-108.

[8] H.M. Kuch, K. Ballschmiter, Determination of endocrine-disrupting phenolic compounds and estrogens in surface and drinking water by HRGC-(NCI)-MS in the picogram per liter range, Environ. Sci. Technol. 35 (2001) 3201-3206.
[9] K.A. Kidd, P.J. Blanchfield, K.H. Mills, V.P. Palace, R.E. Evans, J.M. Lazorchak, R.W. Flick, Collapse of a fish population after exposure to a synthetic estrogen, Proc. Natl. Acad. Sci. USA 104 (2007) 8897-8901.

[10] K. Fent, C. Escher, D. Caminada, Estrogenic activity of pharmaceuticals and pharmaceutical mixtures in a yeast reporter gene system, Reprod. Toxicol. 22 (2006) $175-185$.

[11] J.P. Nash, D.E. Kime, L.T.M. Van der Ven, P.W. Wester, F. Brion, G. Maack P. Stahlschmidt-Allner, C.R. Tyler, Long-term exposure to environmental concentrations of the pharmaceutical ethynylestradiol causes reproductive failure in fish, Environ. Health Perspect. 112 (2004) 1725-1733.

[12] M.J. Ball, E. Ashwell, M.D.G. Gillmer, Progestagen-only oral contraceptives: comparison of the metabolic effects of levonorgestrel and norethisterone, Contraception 44 (1991) 223-233.

[13] R.W. Rebar, K. Zeserson, Characteristics of the new progestogens in combination oral contraceptives, Contraception 44 (1991) 1-10.

[14] E. Vulliet, L. Wiest, R. Baudot, M.-F. Grenier-Loustalot, Multi-residue analysis of steroids at sub-ng/L levels in surface and ground-waters using liquid chromatography coupled to tandem mass spectrometry, J. Chromatogr. A 1210 (2008) 84-91.

[15] L. Viglino, K. Aboulfadl, M. Prévost, S. Sauvé, Analysis of natural and synthetic estrogenic endocrine disruptors in environmental waters using online preconcentration coupled with LC-APPI-MS/MS, Talanta 76 (2008) 1088-1096.

[16] R.A. Bebb, B.D. Anawalt, R.B. Christensen, C.A. Paulsen, W.J. Bremner, A.M. Matsumoto, Combined administration of levonorgestrel and testosterone induces more rapid and effective suppression of spermatogenesis than testosterone alone: a promising male contraceptive approach, J. Clin. Endocrinol. Metab. 81 (1996) 757-762.

[17] M. Guedes Maniero, D. Maia Bila, M. Dezotti, Degradation and estrogenic activity removal of 17 [beta]-estradiol and 17[alpha]-ethinylestradiol by ozonation and $\mathrm{O}_{3} / \mathrm{H}_{2} \mathrm{O}_{2}$, Sci. Total Environ. 407 (2008) 105-115.

[18] Y. Lin, Z. Peng, X. Zhang, Ozonation of estrone, estradiol, diethylstilbestrol in waters, Desalination 249 (2009) 235-240.

[19] X. Zhang, P. Chen, F. Wu, N. Deng, J. Liu, T. Fang, Degradation of 17[alpha]ethinylestradiol in aqueous solution by ozonation, J. Hazard. Mater. 133 (2006) 291-298.

[20] X. Feng, S. Ding, J. Tu, F. Wu, N. Deng, Degradation of estrone in aqueous solution by photo-Fenton system, Sci. Total Environ. 345 (2005) 229-237.

[21] Z. Yaping, H. Jiangyong, Photo-Fenton degradation of 17[beta]-estradiol in presence of [alpha]-FeOOHR and $\mathrm{H}_{2} \mathrm{O}_{2}$, Appl. Catal. B 78 (2008) 250-258.

[22] H.M. Coleman, E.J. Routledge, J.P. Sumpter, B.R. Eggins, J.A. Byrne, Rapid loss of estrogenicity of steroid estrogens by UVA photolysis and photocatalysis over an immobilised titanium dioxide catalyst, Water Res. 38 (2004) 3233-3240.

[23] T. Karpova, S. Preis, J. Kallas, Selective photocatalytic oxidation of steroid estrogens in water treatment: urea as co-pollutant, J. Hazard. Mater. 146 (2007) $465-471$.

[24] T. Karpova, S. Preis, J. Kallas, Selective photocatalytic oxidation of steroid estrogens in the presence of copollutants in the sanitary fraction of domestic sewage, Int. J. Photoenergy 2007 (2007) 1-8.

[25] G. Li Puma, V. Puddu, H.K. Tsang, A. Gora, B. Toepfer, Photocatalytic oxidation of multicomponent mixtures of estrogens (estrone (E1), 17[beta]-estradiol (E2), 17[alpha]-ethynylestradiol (EE2) and estriol (E3)) under UVA and UVC radiation: photon absorption, quantum yields and rate constants independent of photon absorption, Appl. Catal. B 99 (2010) 388-397.

[26] B. Liu, F. Wu, N.-S. Deng, UV-light induced photodegradation of 17[alpha]ethynylestradiol in aqueous solutions, J. Hazard. Mater. 98 (2003) 311-316.

[27] X.L. Liu, F. Wu, N.S. Deng, Photodegradation of 17[alpha]-ethynylestradiol in aqueous solution exposed to a high-pressure mercury lamp (250W), Environ. Pollut. 126 (2003) 393-398.

[28] P. Mazellier, L. Méité, J.D. Laat, Photodegradation of the steroid hormones 17[beta]-estradiol (E2) and 17[alpha]-ethinylestradiol (EE2) in dilute aqueous solution, Chemosphere 73 (2008) 1216-1223.

[29] E.J. Rosenfeldt, P.J. Chen, S. Kullman, K.G. Linden, Destruction of estrogenic activity in water using UV advanced oxidation, Sci. Total Environ. 377 (2007) 105-113.

[30] E.J. Rosenfeldt, K.G. Linden, Degradation of endocrine disrupting chemicals bisphenol A, ethinyl estradiol, and estradiol during UV photolysis and advanced oxidation processes, Environ. Sci. Technol. 38 (2004) 5476-5483.

[31] W. Sun, S. Li, J. Mai, J. Ni, Initial photocatalytic degradation intermediates/pathways of 17[alpha]-ethynylestradiol: effect of $\mathrm{pH}$ and methanol, Chemosphere 81 (2010) 92-99.

[32] P.-J. Chen, E.J. Rosenfeldt, S.W. Kullman, D.E. Hinton, K.G. Linden, Biological assessments of a mixture of endocrine disruptors at environmentally relevant concentrations in water following $\mathrm{UV} / \mathrm{H}_{2} \mathrm{O}_{2}$ oxidation, Sci. Total Environ. 376 (2007) 18-26.

[33] D. Nasuhoglu, V. Yargeau, D. Berk, Photo-removal of sulfamethoxazole (SMX) by photolytic and photocatalytic processes in a batch reactor under UV-C radiation ([lambda]max $=254 \mathrm{~nm}$ ), J. Hazard. Mater. 186 (2011) 67-75.

[34] X. Yang, R.C. Flowers, H.S. Weinberg, P.C. Singer, Occurrence and removal of pharmaceuticals and personal care products (PPCPs) in an advanced wastewater reclamation plant, Water Res. 45 (2011) 5218-5228. 
[35] R. Palominos, J. Freer, M.A. Mondaca, H.D. Mansilla, Evidence for hole participation during the photocatalytic oxidation of the antibiotic flumequine, J. Photochem. Photobiol. A 193 (2008) 139-145.

[36] Y. Chen, S. Yang, K. Wang, L. Lou, Role of primary active species and $\mathrm{TiO}_{2}$ surface characteristic in UV-illuminated photodegradation of Acid Orange 7, J. Photochem. Photobiol. A 172 (2005) 47-54.
[37] N.J. Bunce, J. Lamarre, S.P. Vaish, Photorearrangement of azoxybenzene to 2-hdroxyazobenzene: a convenient chemical actinometer, Photochem. Photobiol. 39 (1984) 531-533.

[38] V.K. Gupta, R. Jain, A. Nayak, S. Agarwal, M. Shrivastava, Removal of the hazardous dye - tartrazine by photodegradation on titanium dioxide surface, Mat. Sci. Eng. C 31 (2011) 1062-1067. 